\title{
The young stellar population at the center of NGC 205
}

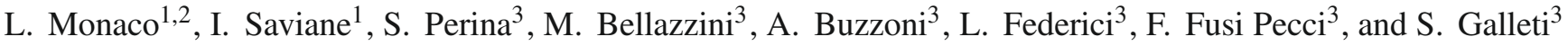 \\ 1 European Southern Observatory, Casilla 19001, Santiago, Chile \\ e-mail: lmonaco@astro-udec.cl \\ 2 Universidad de Concepción, Casilla 160-C, Concepción, Chile \\ 3 Istituto Nazionale di Astrofisica - Osservatorio Astronomico di Bologna, 40127 Bologna, Italy \\ Received 2 May 2009 / Accepted 22 June 2009
}

\section{ABSTRACT}

\begin{abstract}
Context. NGC 205 is a peculiar dwarf elliptical galaxy hosting in its center a population of young blue stars. Their origin is still matter of debate, the central fresh star formation activity possibly being related to dynamical interactions between NGC 205 and M 31.

Aims. The star formation history in the central $30^{\prime \prime}(\sim 120 \mathrm{pc})$ around the NGC 205 central nucleus is investigated in order to obtain clues to the origin of the young stellar population.

Methods. Deep HST/ACS CCD photometry is compared with theoretical isochrones and luminosity functions to characterize the stellar content of the region under study and compute the recent SF rate.

Results. Our photometry reveals a previously undetected blue plume of young stars clearly distinguishable down to $I \simeq 26$. Our analysis suggests that $1.9 \times 10^{5} M_{\odot}$ were produced between approximately $62 \mathrm{Myr}$ and $335 \mathrm{Myr}$ ago in the NGC 205 inner regions, with a latest minor episode occurring $\sim 25 \mathrm{Myr}$ ago. This implies a star formation rate of $\sim 7 \times 10^{-4} M_{\odot} /$ yr over this period. Conclusions. The excellent fit of the observed luminosity function of young main sequence stars obtained with a model having a constant star formation rate argues against a tidally triggered star formation activity over the last $\sim 300$ Myr. Rather, a constant SF may be consistent with NGC 205 being on its first interaction with M 31.
\end{abstract}

Key words. galaxies: evolution - galaxies: dwarf - galaxies: individual: NGC $205 \mathrm{dE}$

\section{Introduction}

NGG 205 is one of the brightest $\left(M_{V}=-16.6\right.$ Mateo 1998) M 31 satellites and is a peculiar nucleated dwarf elliptical galaxy. It hosts a fairly complex stellar content. Color-magnitude diagrams and time series revealed the presence in NGC 205 of RR Lyrae and carbon stars, i.e. both old and intermediate age stars as well as sequences of asymptotic and red giant branch stars (AGB/RGB, Sharina et al. 2006; Richer et al. 1984; Davidge 2003, 2005). Lee (1996) broadly summarized the NGC 205 star formation (SF) history.

The presence of a population of bright blue stars in the most central region of NGC 205 has been known since the early studies (Hodge 1973, and references therein) on this galaxy and is an unusual characteristic for a dwarf elliptical ${ }^{1}$. Cappellari et al. (1999, hereafter C99) revealed that many of the brightest blue sources classified as very young stars (Peletier 1993) were in fact multiple systems, clusters or star associations. Davidge (2003, hereafter D03), studying the population of Asymptotic Giant Branch (AGB) stars, noted that multiple episodes of SF may have occurred in the most central regions (see also Bica et al. 1990; Lee 1996) with a time spacing compatible with the putative NGC 205 orbital period (Cepa \& Beckman 1988). Therefore, tidal interactions with M 31 could have triggered the latest episodes of SF. Indeed, evidence of past dynamical interactions of NGC 205 with its bright companion has been accumulating over the years (McConnachie et al. 2004; Geha et al. 2006; De Rijcke et al. 2006) and residual gas (Welch et al. 1998) and

\footnotetext{
1 On the other hand, young nuclear clusters seem relatively frequent in nucleated dwarfs (Rossa et al. 2006).
}

dust (Marleau et al. 2006) have also been detected in NGC 205 (see also Davidge 2005).

However, in spite of the great interest in the recent star formation history in the innermost region of this galaxy, previous attempts to directly analyze the young Main Sequence population have been unsuccessful, likely due to the extreme degree of crowding affecting the region of interest (Butler \& Martínez-Delgado 2005). Here we use the exceptional spatial resolution of the Advanced Camera for Survey (ACS) on board the Hubble Space Telescope (HST) to derive accurate stellar photometry in the circum-nuclear region of NGC 205, resolving for the first time the young Main Sequence (MS) population down to $I \simeq 26$.

\section{Data reduction}

The inner $29^{\prime \prime} \times 26^{\prime \prime}$ around the central nucleus of NGC 205 were imaged using the ACS high resolution channel (HRC) and retrieved from the archive through the MAST web interface ${ }^{2}$. At the distance of NGC 205 (824 kpc, see McConnachie et al. 2005), the observed field of view corresponds to about $120 \mathrm{pc}$. The data were obtained during Cycle 11 (program 9448, PI L. Ferrarese). $F 555 W$ and $F 814 W$ frames were taken for a total integration time of $2560 \mathrm{~s}$ and $2440 \mathrm{~s}$, respectively. Images were acquired at four different pointings to improve the resolution and a pixel scale of $0.022 \mathrm{px}^{-1}$ was eventually obtained (see Valluri et al. 2005, for further details). Photometry was performed using the ACS module of Dolphot ${ }^{3}$, with the same approach described

\footnotetext{
2 http://archive.stsci.edu/

3 http://purcell.as.arizona.edu/dolphot/
} 
in Galleti et al. (2006) and Perina et al. (2009). A final photometric catalog of about 26000 stars was built, retaining only objects classified as bona-fide stars (i.e., quality flag $=1$ ) and having a $\chi^{2}<2.5$. This selection allows us to exclude several spurious and/or poorly measured sources without a significant loss of information (Galleti et al. 2006). Dolphot automatically transforms instrumental magnitudes into the VEGAMAG and the Johnson-Kron-Cousins BVRI systems adopting the calibrations by Sirianni et al. (2005). In the following we will always adopt Johnson-Kron-Cousins $V$, I magnitudes.

The majority of the central nucleus is unresolved and stars begin to be measured at radial distances $\geq 00^{\prime} 9$ (about $40 \mathrm{px}$ ) from the cluster center. At a radius of $\sim 5^{\prime \prime}$, the nucleus surface brightness profile joins the profile of the underlying galaxy (see Valluri et al. 2005; Butler \& Martínez-Delgado 2005; Jones et al. 1996). There is no particular difference in the stellar population in the annulus $1^{\prime \prime} \lesssim r \lesssim 5^{\prime \prime}$, where the resolved outskirts of the nuclear cluster are mixed with the underlying population, and the surrounding field. Therefore, in the following, we will consider the whole sample as representative of the circum-nuclear region of the galaxy.

\subsection{Comparison with previous photometries}

Figure 1 shows the obtained $I$ vs. $V-I$ color-magnitude diagram (CMD). WFPC2 photometry of the central region of NGC 205 was presented by Butler \& Martínez-Delgado (2005, hereafter BM05, their fields F3 and F4). An inspection of Fig. 1 and their Fig. 4 reveals that the ACS photometry presented here is significantly deeper than any other obtained so far for the same field. Also, evolutionary marks like the clump of stars at $V-I \simeq 0.9$; $I \simeq 21.8$ (hereafter YC, young clump, see Sect. 3) have never been detected before.

While the quality of the WFPC2 and ACS photometries are comparable along the RGB ( $V-I \geq 1)$, the populated young MS (blue plume, BP) visible in Fig. 1 at $V-I \simeq-0.2$ down to the detection limit is practically absent in WFPC2 CMDs. Quite likely, such blue stars do not exist at all in the more external regions of NGC 205. However, they could have been detected in the F3 and F4 fields, which encircle the ACS area.

Blending of red stars may appear in the photometry as sequences of spurious blue stars. Extensive artificial star tests have been performed following the procedure described in Perina et al. (2009) to assess the completeness and the impact of blending on our photometry as a function of the star color and magnitude. The bin-migration effect is quite limited for stars having $V-I<0.5$. In the magnitude range $22<I<24.5$, only a fraction between $2 \%$ and $4.5 \%$ of the injected stars change their magnitude by $\Delta I \geq-0.5$ when recovered. This means that about 64 in the $\sim 1764$ stars under consideration $(\sim 3.6 \%)$ may be seriously affected by blending of star pairs. We are thus fully confident about the genuine nature of the detected young MS and that blending effects do not jeopardize the results presented. The lack of such BP in the BM05 photometry is likely to be due to the details of the photometry process and to the selection criteria adopted to filter the detected sources.

It has long been known that the brightest blue stars in NGC 205 are concentrated in a region of $\sim 300 \mathrm{pc}\left(100^{\prime \prime}\right)$ around the nucleus (Hodge 1973). Several authors have presented integrated photometry surface brightness profiles over extended areas in NGC 205 (see Lee 1996, and references therein). The galaxy colors become bluer inward within $\sim 50^{\prime \prime}$ from the center with the exception of the inner $\sim 2^{\prime \prime}$ where the colors become redder. Most of the bright blue stars were found to be

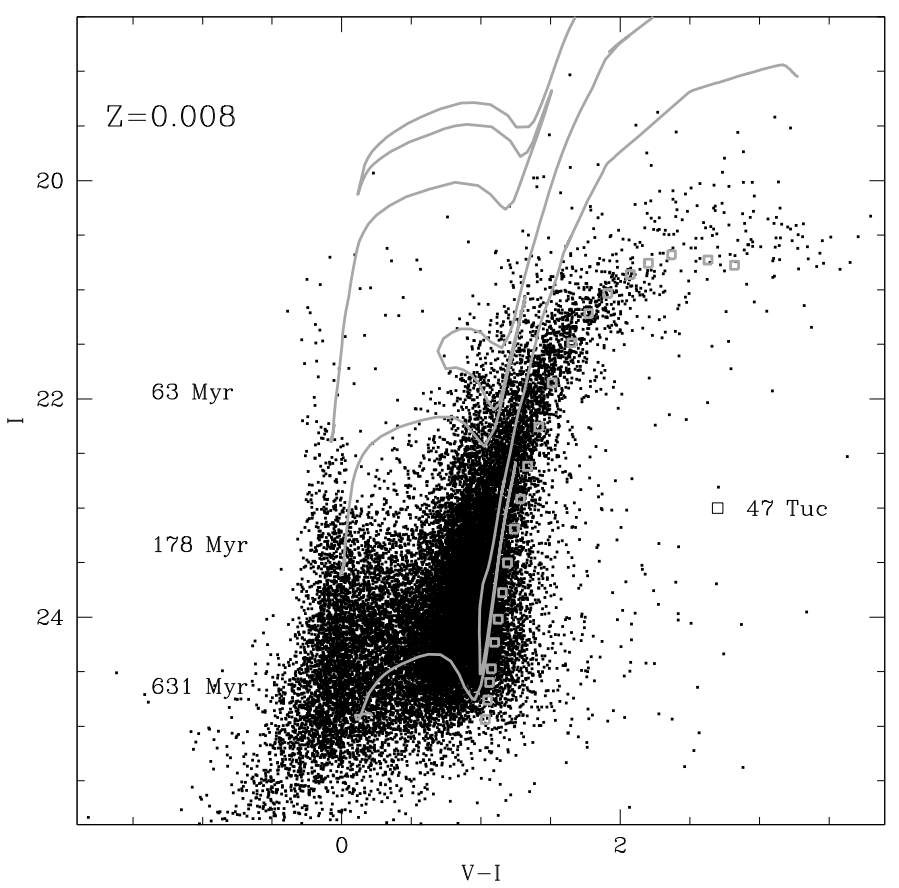

Fig. 1. Theoretical post MS isochrones $(Z=0.008)$ from the Girardi et al. (2002) library superimposed on the ACS photometry. The mean ridge line of the galactic globular cluster 47 Tucanae is also plotted as open squares.

concentrated within a region of $\sim 20^{\prime \prime}$ diameter around the central nucleus (Peletier 1993). However, as already mentioned, many of these stars were in fact clusters or star associations, as pointed out by C99 using WFPC2 data.

The spatial distribution of BP stars detected in our ACS photometry, on the other hand, does not present any obvious clustering, i.e. they do not belong to associations similar to the ones discovered by C99 (which are located just outside the limits of our ACS field). The same applies also to stars belonging to the YC.

\section{Star formation history}

An overall idea of the star formation history in the observed NGC 205 regions can be readily obtained with the aid of theoretical isochrones. On top of the ACS photometry, we plotted in Fig. 1 a selection of isochrones (in the post MS phase) from the Girardi et al. (2002) library and the mean ridge line of the galactic globular cluster 47 Tucanae (open squares) from Saviane et al. (2000). Reddening and distances for this cluster were taken from the most recent version of the Harris (1996) catalogue.

We adopted for NGC 205 the following parameters: $(m-$ $M)_{0}=24.58, E(B-V)=0.08$ from McConnachie et al. (2005) and Schlegel et al. (1998), respectively. However, this reddening value does not account for the internal extinction nor for the contribution from M31's dust clouds in front of NGC 205. While it has been suggested that NGC 205 might even lie in front of M 31 (Howley et al. 2008), some degree of differential reddening is certainly present in the galaxy region under consideration. Nevertheless, its overall effect on the photometry presented here is negligible (see BM05 for a discussion).

Figure 1 shows that the blue plume $(V-I \simeq-0.2)$ is dominated by MS stars likely older than 50-60 Myr and reaching $\sim 650 \mathrm{Myr}$ at the detection limit. A sparse population of younger 
stars is also visible at $I \leq 22$. They might correspond to a $\sim 25$ Myr old simple stellar population (see below). The YC may mark instead a star formation episode occurring some $\sim 200 \mathrm{Myr}$ ago and is made up of blue-loop helium burning stars. The bulk of the RGB population is matched at its red edge by the ridge line of the old and relatively metal rich globular cluster 47 Tuc $(\mathrm{Fe} / \mathrm{H}=-0.70$, Saviane et al. 2000), and is broadly compatible with $Z=0.008$ isochrones a few Gyr old. Sharina et al. (2006), using the Lick indexes, derived a mean metallicity of $[\mathrm{Z} / \mathrm{H}] \simeq-0.5$, age of $1.9 \mathrm{Gyr}$ and no alpha-enhancement for their most central field outside the nucleus (see also Mould et al. 1984, BM05). This is also consistent with the presence of a well populated red-clump along the RGB at $I>24$ (see also BM05) which, at odds with the BP population, is a dominant feature also in the CMDs of external regions of NGC 205 (see BM05).

\subsection{Recent star formation history}

The luminosity function (LF) of the MS can be used to derive the mass in young stars and the star formation rate $(S F R)$ at late epochs in the central region of NGC 205 (see Saviane et al. 2004, hereafter SHR04). We assume for the moment that the SF has been continuous and constant (but see next section) for the last few hundred Myr. Thus, the overall SF activity can be approximated by the sum of a discrete number of simple stellar populations (SSPs). Each generation of stars will produce a power law LF which is added to that of the previous generations. The observed LF will result, then, from the convolution of the LFs of the SSPs (see SHR04).

We select BP stars in the color region at $V-I<0.2$. Besides MS stars, a negligible fraction of blue loop stars may also be present in the selected sample. In the color range under consideration, the completeness factor is $\sim 0.66$ at $I=24.5$ and a comparison of the surface brightness profile presented by Valluri et al. (2005) with a star-count based one reveals that no radial variations of the completeness factor are present for $I \leq 24.5$, as long as radial distances greater then $r>55^{\prime \prime} 1$ from the central nucleus are considered. Therefore, in the following, only stars having $I \leq 24.5$ (i.e. $M_{V} \leq-0.5$ ) and $r>5$ '. 1 will be included in the analysis. Figure 2 shows the logarithmic cumulated LF of the selected stars (diamonds), corrected for the appropriate completeness factors.

In order to estimate the mass in young stars, we followed the same approach as SHR04 (see lower panel in Fig. 2). For stars fainter than $M_{V} \simeq-3.0$ (i.e. $I \simeq 22$ ), the observed LF is well reproduced (heavy solid line) by the sum of 20 LFs of simple stellar populations (dot-dashed lines). The slope of the single LFs is assumed equal to that of the young Large Magellanic Cloud cluster NGC 2004 (see SHR04 for details). Each SSP contains half of the stars in NGC 2004. The populations were generated at a constant rate, and all stars now evolved off the main sequence were removed from the LFs. This way we obtain that $\sim 1.9 \times 10^{5} M_{\odot}$ were produced between $\sim 62 \mathrm{Myr}$ and $\sim 335 \mathrm{Myr}$ ago. Therefore, the star formation rate over this $\sim 273$ Myr lapse has been $\sim 7 \times 10^{-4} M_{\odot} / y r$. Note, however, that the star formation should have been active since at least $\sim 650 \mathrm{Myr}$ ago (see Fig. 1).

The sparse population of stars brighter than $I \simeq 22\left(M_{V}<\right.$ -3) presents instead a flatter LF which may be compatible with an isolated SF episode. The age of this population can be estimated by assuming that the brightest stars are near the termination of the MS. Using formula B1 of SHR04, their absolute luminosity $\left(M_{V} \simeq-4.2\right)$ yields an age of $\sim 25 \mathrm{Myr}$. Hence, this population would be slightly younger than the clusters studied by C99 (50 Myr and 100 Myr, respectively).

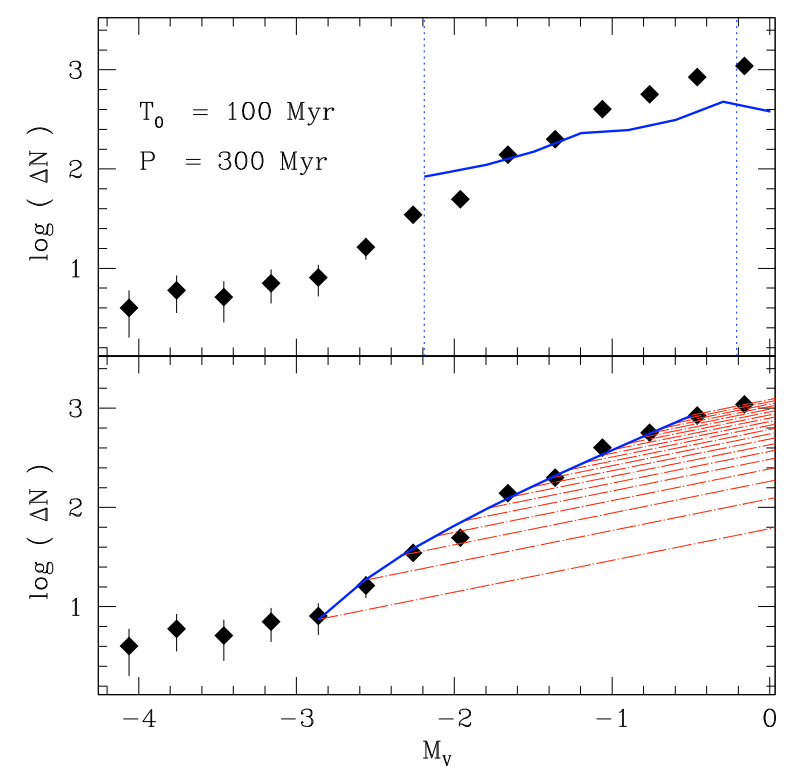

Fig. 2. Lower panel: logarithmic integrated LF of young MS stars (filled diamonds). The LF is reproduced by the sum (thick continuous line) of $20 \mathrm{LFs}$ (dot-dashed lines) of simple stellar populations. It is assumed here that the slope of the single LFs is equal to that of NGC 2004 (a young populous cluster of the Large Magellanic Cloud; $\alpha=0.32 \pm 0.04$, see Fig. 13 in SHR04) and that the simple stellar population contains 0.5 times the number of stars in NGC 2004. The populations were generated at a constant rate during $\sim 273 \mathrm{Myr}$ (see Sect. 3.1), and all stars now evolved off the main sequence were removed from the LFs. Upper panel: the observed LF (filled diamonds) compared with a synthetic one (thick continuous line) generated with a bursting star formation. The epoch of the last episode $\left(T_{0}\right)$ and the time lapse between the star formation episodes (P) are indicated. Dotted vertical lines mark the termination of the MS for the single bursts. See SHR04 and text for details.

Relying on the Burstein et al. (1988, hereafter B88) IUE data, the UV flux at $1550 \AA$ leads to a galaxy luminosity of $\log L_{1550}=23.63 \mathrm{ergs} \mathrm{s}^{-1} \mathrm{~Hz}^{-1}$ across the IUE field of view. Such a luminosity corresponds to a current $S F R \lesssim 10^{-5} M_{\odot} \mathrm{yr}^{-1}$, according to the Buzzoni (2002) calibration. The IUE data indicate, therefore, a drastic reduction of galaxy SF at the current epoch.

Based on a further set of IUE observation, that sampled the nucleus of NGC 205 (region "N"), and the region 1' north of it (region "B"), Wilcots et al. (1990) estimated a mass of young stars $\left(M \geq 1 M_{\odot}\right)$ of 2 and $1 \times 10^{5} M_{\odot}$ for the two galaxy regions, respectively. Region B corresponds to a part of NGC 205 where some bright blue stars were observed but does not overlap with our field. Their region $\mathrm{N}$ value and ours are similar. However, their value includes the contribution from the central nucleus which, instead, is excluded in our LF-based estimate. Furthermore, they estimate the mass of all stars having $M \geq 1 M_{\odot}$, while we neglect the contribution by star formation episodes older than $335 \mathrm{Myr}$, as they do not show up in the LF in the selected magnitude range (see Fig. 2). On the other hand, the IUE field of view is equivalent to a circular aperture with a diameter of 14" (see B88), and, as such, it corresponds to about a fifth of the observed ACS field.

\section{Summary and discussion}

We presented new, deep ACS photometry of the inner 30" $(\sim 120 \mathrm{pc})$ around the NGC 205 nucleus. The most notable 
feature presented here is the well populated blue plume of young MS stars visible at $V-I<0.2$ down to the detection limit $(I \simeq 26$, see Fig. 1). Previous photometries were unable to probe this population, which is confined to the central galaxy regions. The LF of blue plume stars has been used to investigate the recent star formation in the central region of NGC 205. We found that $\sim 1.9 \times 10^{5} M_{\odot}$ were produced between $\sim 62 \mathrm{Myr}$ and $\sim 335 \mathrm{Myr}$ ago, corresponding to a star formation rate of $\sim 7 \times 10^{-4} M_{\odot} /$ yr. However, star formation has been active since at least $\sim 650 \mathrm{Myr}$ ago, which corresponds to our detection limit (see Fig. 1). A small number of $\sim 25 \mathrm{Myr}$ old stars are also present, in agreement with previous findings by C99 and Lee (1996).

BM05 and D03 concluded that the latest star formation episodes occurred a few $10^{8} \mathrm{yr}$ ago in NGC 205. This led them both to speculate that the recent star formation in NGC 205 might have been triggered by past interactions with $M$ 31. This is certainly an intriguing possibility. In fact, Cepa \& Beckman (1988, hereafter CB88) estimated the orbital period of NGC 205 in $300 \mathrm{Myr}$, with the last passage through the M31 disk occurring 100 Myr ago. However, the LF of the young MS is compatible with a constant SF rate, at least over the last $\sim 300 \mathrm{Myr}$ (see Fig. 2, lower panel).

In Fig. 2 (upper panel), we compare the young NGC 205 MS LF (filled diamonds) to the LF expected in the presence of a bursting star formation activity (thick continuous line). The BP mean ridge line and its broadening were adopted to generate a synthetic MS. For each SF episode, the termination of the MS was determined using formula B1 in SHR04. Each burst is assumed to produce the same number of stars and an NGC 2004like LF. The resultant - synthetic - LF is the sum of the stars generated in the single bursts, normalized to the total number of observed stars.

We assumed bursts to occur with a time spacing equal to the orbital period $(P=300 \mathrm{Myr})$, the last SF episode having occurred at the epoch of the last passage, $T_{0}=100 \mathrm{Myr}$ ago. The vertical lines mark the termination of the MS of the various SF episodes. Given the above parameters, most of the magnitude range under consideration is covered by just one episode of SF (see Fig. 1) and the synthetic LF is clearly not compatible with the observed one. More in general, a bursting SF with a period longer than $\sim 100$ Myr would imply an LF flatter than observed. On the other hand, a series of closely spaced star formation episodes would approximate a continuous star formation (lower panel) and, as expected, we find that the observed LF is well reproduced by a series of episodes spaced by only 10-20 Myr, the last one occurring $\sim 63$ Myr ago (see also Fig. 1). This kind of activity is reminiscent of the stochastic self-propagating star formation theory proposed by Gerola \& Seiden (1978) and later discussed, e.g., by Shore (1983) and Valle et al. (2005).

Furthermore, the star formation has certainly been active since more than $\sim 650$ Myr ago (see Fig. 1) and no indication of a significantly enhanced SF activity at any particular epoch is detected. The only feature that may be connected to an enhancement of the SF rate over a timescale comparable with the expected orbital period is the YC, that is presumably associated with a short episode that occurred 200 Myr ago. Therefore, our photometry does not lend support the hypothesis of a tidally triggered SF at late epochs in NGC 205. Rather, a continuous SF may be consistent with NGC 205 being in its first interaction with M 31, as recently proposed by Howley et al. (2008).

The presence of a young population in a dwarf elliptical (over spatial scales much larger than the nucleus) remains puzzling, as is the significant amount of gas observed in this galaxy.
The detected amount of gas, however, seems to be compatible with being returned to the interstellar medium by evolved stars during a burst of star formation starting $\sim 5 \times 10^{8} \mathrm{yr}$ ago (Marleau et al. 2006). The present letter shows that the SF was indeed active on a similar time scale.

Lisker et al. (2006) discovered a number of dE with disklike features in the Virgo cluster and introduced the term "dEdi" for those galaxies. Their analysis supports the idea that dEdis is a population of genuine disk galaxies and not just spheroids hosting a disk, perhaps only of tidal origin. These authors also flag NGC 205 as dEbc type, a subclass of the dEdis having blue centers (see Lisker et al. 2007). In fact, the large scale dynamics reveals that NGC 205 is at least partly supported by rotation (De Rijcke et al. 2006; Geha et al. 2006), with rotation being detected only along the major axis and De Rijcke et al. (2006) inferred an oblate geometry for the galaxy mass distribution. Also, recently obtained ground-based images provide compelling evidence that NGC 205 indeed hosts an embedded stellar disk (Saviane et al., in preparation). A revised classification from $\mathrm{dE}$ to a disk galaxy would provide a natural explanation for many of the NGC 205 peculiarities, including the presence of gas and the continuous star formation occurring in its central regions during (at least) the last $\sim 650 \mathrm{Myr}$, as revealed by the present study.

Acknowledgements. M.B. and S.P acknowledge the financial support of INAF through the PRIN 2007 grant CRA 1.06.10.04.

\section{References}

Bica, E., Alloin, D., \& Schmidt, A. A. 1990, A\&A, 228, 23 Burstein, D., Bertola, F., Buson, L. M., et al. 1988, ApJ, 328, 440 (B88) Butler, D. J., \& Martínez-Delgado, D. 2005, AJ, 129, 2217 (BM05) Buzzoni, A. 2002, AJ, 123, 1188

Cappellari, M., Bertola, F., Burstein, D., et al. 1999, ApJ, 515, L17 (C99)

Cepa, J., \& Beckman, J. E. 1988, A\&A, 200, 21 (CP88)

Davidge, T. J. 2003, ApJ, 597, 289 (D03)

Davidge, T. J. 2005, AJ, 130, 2087

De Rijcke, S., Prugniel, P., Simien, F., et al. 2006, MNRAS, 369, 1321

Galleti, S., Federici, L., Bellazzini, M., et al. 2006, ApJ, 650, L107

Geha, M., Guhathakurta, P., Rich, R. M., \& Cooper, M. C. 2006, AJ, 131, 332

Gerola, H., \& Seiden, P. E. 1978, ApJ, 223, 129

Girardi, L., Bertelli, G., Bressan, A., et al. 2002, A\&A, 391, 195

Harris, W. E. 1996, AJ, 112, 1487

Hodge, P. W. 1973, ApJ, 182, 671

Howley, K. M., Geha, M., Guhathakurta, P., et al. 2008, ApJ, 683, 722

Jones, D. H., Mould, J. R., Watson, A. M., et al. 1996, ApJ, 466, 742

Lee, M. G. 1996, AJ, 112, 1438

Lisker, T., Grebel, E. K., \& Binggeli, B. 2006, AJ, 132, 497

Lisker, T., Grebel, E. K., Binggeli, B., \& Glatt, K. 2007, ApJ, 660, 1186

Marleau, F. R., Noriega-Crespo, A., Misselt, K. A., et al. 2006, ApJ, 646, 929

Mateo, M. L. 1998, ARA\&A, 36, 435

McConnachie, A. W., Irwin, M. J., Lewis, G. F., et al. 2004, MNRAS, 351, L94

McConnachie, A. W., Irwin, M. J., Ferguson, A. M. N., et al. 2005, MNRAS, 356,979

Mould, J., Kristian, J., \& Da Costa, G. S. 1984, ApJ, 278, 575

Peletier, R. F. 1993, A\&A, 271, 51

Perina, S., Barmby, P., Beasley, M. A., et al. 2009, A\&A, 494, 933

Richer, H. B., Crabtree, D. R., \& Pritchet, C. J. 1984, ApJ, 287, 138

Rossa, J., van der Marel, R. P., Böker, T., et al. 2006, PASP, 109, 907

Saviane, I., Rosenberg, A., Piotto, G., \& Aparicio, A. 2000, A\&A, 355, 966

Saviane, I., Hibbard, J. E., Rich, R. M. 2004, AJ, 127, 660 (SHR04)

Schlegel, D. J., Finkbeiner, D. P., \& Davis, M. 1998, ApJ, 500, 525

Sharina, M. E., Afanasiev, V. L., \& Puzia, T. H. 2006, MNRAS, 372, 1259

Shore, S. N. 1983, ApJ, 265, 202

Sirianni, M., Jee, M.J., Bemitez, N., et al. 2005, PASP, 117, 1049

Valle, G., Cignoni, M., \& Shore, S. N. 2005, A\&A, 440, 473

Valluri, M., Ferrarese, L., Merritt, D., \& Joseph, C. L. 2005, ApJ, 628, 137

Welch, G. A., Sage, L. J., \& Mitchell, G. F. 1998, ApJ, 499, 209

Wilcots, E. M., Hodge, P., Eskridge, P. B., et al. 1990, ApJ, 364, 87 